\title{
IRREDUCIBLE REPRESENTATIONS OF THE GENERALIZED SYMMETRIC GROUP $B_{n}^{m}$
}

\author{
by M. SAEED-UL-ISLAM
}

(Received 2 July, 1984; revised 4 May, 1986)

Introduction. This paper is devoted to the determining of the irreducible linear representations of the generalized symmetric group $B_{n}^{m}$ (elsewhere written as $C_{m}^{n} S_{n}$, $C_{m}\left\{S_{n}\right.$ or $\left.G(m, 1, n)\right)$ by considering the conjugacy classes of $B_{n}^{m}$ and then constructing the same number of inequivalent irreducible linear representations of $B_{n}^{m}$. These have previously been determined by Kerber [2, Section 5] using Clifford's theory applied to wreath products.

An independent approach is given here which does not use Clifford's theory, and some of the results of Kerber [2], Puttaswamaiah [4] and Osima [3] are obtained in a much easier and more elementary way. The analogous problem of determining the irreducible projective representations of the generalized symmetric group has been treated in [6].

Elementary knowledge of representation theory is assumed. The symbol $\mathbb{C}^{*}$ will denote the multiplicative group of non-zero complex numbers, $\mathbb{N}$ the set of natural numbers and $\mathbb{N}^{*}=\mathbb{N} \cup\{0\}$.

2. The group $B_{n}^{m}$ and its conjugacy classes. A set of generators and relations for $B_{n}^{m}$ is given by

$$
\begin{aligned}
& B_{n}^{m}=\left\{r_{1}, \ldots, r_{n}: r_{i}^{2}=1=r_{n}^{m}, i=1, \ldots, n-1 ;\left(r_{i} r_{i+1}\right)^{3}=1, i=1, \ldots, n-2 ;\right. \\
& \left.\left(r_{n-1} r_{n}\right)^{2}=\left(r_{n} r_{n-1}\right)^{2},\left(r_{i} r_{j}\right)^{2}=1, i, j=1, \ldots, n, j \neq i, i+1\right\}
\end{aligned}
$$

(see Coxeter [1]).

We may identify $r_{i}(i=1, \ldots, n-1)$ with the transposition $(i, i+1)$ and therefore the group generated by $r_{1}, \ldots, r_{n-1}$ is the symmetric group $S_{n}$.

The generator $r_{n}$ may be identified with the mapping

defined by

$$
\left(\begin{array}{c}
n \\
\xi_{n}
\end{array}\right):\{1, \ldots, n\} \rightarrow \mathbb{C}^{*}
$$

$$
j \rightarrow j, j=1, \ldots, n-1 \text { and } n \rightarrow \xi n,
$$

where $\xi$ is some primitive $m$ th root of unity.

Consequently an element

$$
r_{i} \ldots r_{n-1} r_{n} r_{n-1} \ldots r_{i}, i=1, \ldots, n-1
$$

corresponds to the mapping

$$
\left(\begin{array}{c}
i \\
\xi_{i}
\end{array}\right):\{1, \ldots, n\} \rightarrow \mathbb{C}^{*}
$$

Glasgow Math. J. 29 (1987) 1-6. 
defined by

$$
j \rightarrow j, j=1, \ldots, i-1, i+1, \ldots, n \text { and } i \rightarrow \xi i .
$$

An arbitrary element $\sigma \in B_{n}^{m}$ may be expressed uniquely as the product of disjoint cycles $\sigma=\theta_{1} \ldots \theta_{t}$, where

$$
\theta_{i}=\left(\begin{array}{cccc}
b_{i 1} & b_{i 2} & \cdots & b_{i i_{i}} \\
\xi^{k_{i 1}} b_{i 2} & \xi^{k_{i 2}} b_{i 3} & \cdots & \xi^{k_{i i}} b_{i 1}
\end{array}\right),
$$

$b_{i j} \in\{1, \ldots, n\}, k_{i j} \in\{1, \ldots, m\}$ and $t_{i}$ is the length of the cycle $\theta_{i}, i=1, \ldots, t$. (See Read [5] for more details.)

Definition 2.1. Let $\sigma=\theta_{1} \ldots \theta_{t}$ as above. Define $f\left(\theta_{i}\right)=\sum_{j=1}^{t_{i}} k_{i j}$ and put $f(\sigma)=$ $\sum_{i=1}^{t} f\left(\theta_{i}\right)$. Let $a_{r s}(\sigma)$ denote the number of cycles $\theta_{i}$ of $\sigma$ such that $f\left(\theta_{i}\right) \equiv r(\bmod m)$, $1 \leqq r \leqq m, 1 \leqq s \leqq n$. Then the $m \times n$ matrix $\left(a_{r s}(\sigma)\right)$ is called the type of $\sigma$, and will be written as type $(\sigma)$.

LemMA 2.2. Two elements $\sigma$ and $\sigma^{\prime}$ of $B_{n}^{m}$ are conjugate if and only if type $(\sigma)=$ $\operatorname{type}\left(\sigma^{\prime}\right)$.

Proof. See Kerber $[2,3.7]$.

Lemma 2.3. Let $t_{i} \in \mathbb{N}^{*}$ and let $p\left(t_{i}\right)$ be the number of partitions of $t_{i}$ if $t_{i} \in \mathbb{N}$ and $p(0)=1$. Then the number of conjugacy classes of $B_{n}^{m}$ is given by

$$
\sum p\left(t_{1}\right) \ldots p\left(t_{m}\right)
$$

where the summation is taken over all the $m$-tuples $\left(t_{1}, \ldots, t_{m}\right)$ such that $\sum_{i=1}^{m} t_{i}=n$.

Proof. We prove the lemma by establishing a one-to-one correspondence between the set of all the conjugacy classes of $B_{n}^{m}$ and the set of all the $m$-partitions of $n$. By an $m$-partition of $n$ we mean an $m$-tuple $\left(\pi\left(t_{1}\right), \ldots, \pi\left(t_{m}\right)\right), t_{i} \in \mathbb{N}^{*}$ such that $t_{1}+\ldots+t_{m}=$ $n$ and each $\pi\left(t_{i}\right)$ is an $n$-tuple $\left(a_{i 1}, \ldots, a_{i n}\right)$, where $a_{i j} \in \mathbb{N}^{*}$ and $\sum_{j=1}^{n} j a_{i j}=t_{i}$.

If a conjugacy class is of type $\left(a_{i j}\right)$, we associate with it an $m$-partition given by $\left(\pi\left(t_{1}\right), \ldots, \pi\left(t_{m}\right)\right)$, where $t_{i}=\sum_{j=1}^{n} j a_{i j}$ and $\pi\left(t_{i}\right)=\left(a_{i 1}, \ldots, a_{i n}\right)$. Clearly, this partition is uniquely defined and conjugacy classes of different types correspond to different $m$-partitions of $n$.

Conversely, let $\left(\pi\left(t_{1}\right), \ldots, \pi\left(t_{m}\right)\right)$ be an $m$-partition of $n$, where $\pi\left(t_{i}\right)=$ $\left(a_{i 1}, \ldots, a_{i n}\right)$. Then it can be easily seen that the set $\{1, \ldots, n\}$ can be uniquely expressed as a disjoint union of $\sum_{i, j} a_{i j}$ subsets such that exactly $\sum_{i=1}^{n} a_{i j}$ of these subsets have $j$ 
elements. On each of these subsets define a cyclic permutation as follows. For example, if $A=\left\{b_{1}, \ldots, b_{j}\right\}$ is one of the subsets having $j$ elements, define

$$
\theta_{A}=\left(\begin{array}{cccc}
b_{1} & b_{2} & \cdots & b_{j} \\
b_{2} & b_{3} & \cdots & \xi^{k} b_{1}
\end{array}\right)
$$

where $k=1$ for the first $a_{1 j}$ subsets having $j$ elements, $k=2$ for the next $a_{2 j}$ subsets having $j$ elements and so on. Let $\sigma$ be the product of all such cycles. Clearly type $(\sigma)=\left(a_{i j}\right)$. This completes the proof.

\section{Generalized Young subgroups and basic representations.}

Definition 3.1. Let $\left(t_{1}, \ldots, t_{k}\right)$ be a $k$-tuple such that $t_{i} \in\{0,1, \ldots, n\}$ and $t_{1}+\ldots+t_{k}=n$. We shall call $\left(t_{1}, \ldots, t_{k}\right)$ a permissible $k$-tuple. Define $p_{0}=0$ and $p_{i}=\sum_{j=1}^{i} t_{j}, i=1, \ldots, k$. If $t_{i} \neq 0$, let $B_{t_{i}}^{m}$ be the generalized symmetric group on the $t_{i}$ symbols

$$
P_{i}=\left\{p_{i-1}+1, \ldots, p_{i}\right\}, i=1, \ldots, k,
$$

and let $B_{0}^{m}=1$, the trivial subgroup of $B_{n}^{m}$.

The group $B_{t_{1}}^{m} \times B_{t_{2}}^{m} \times \ldots \times B_{t_{k}}^{m}$ is called the generalized Young subgroup determined by the $k$-tuple $\left(t_{1}, \ldots, t_{k}\right)$. We denote this group by $B_{\left(t_{1}, \ldots, t_{k}\right)}^{m}$.

Let

$$
\sigma=\left(\begin{array}{cccc}
1 & 2 & \ldots & n \\
\xi^{k_{1}} b_{1} & \xi^{k_{2}} b_{2} & \ldots & \xi^{k_{n}} b_{n}
\end{array}\right) \in B_{n}^{m}
$$

where $b_{1}, \ldots, b_{n} \in\{1, \ldots, n\}$ and the $k_{i}$ are positive integers. Define $\phi: B_{n}^{m} \rightarrow S_{n}$ by

$$
\phi(\sigma)=\left(\begin{array}{cccc}
1 & 2 & \ldots & n \\
b_{1} & b_{2} & \ldots & b_{n}
\end{array}\right) .
$$

Then $\phi$ is an epimorphism and it can be verified by induction on $i$ that the kernel of $\phi$ is an abelian group generated by

$$
\left\{r_{i} \ldots r_{n-1} r_{n} r_{n-1} \ldots r_{i}: i=1, \ldots, n\right\} .
$$

The kernel of $\phi$ is in fact the direct product of $n$ cyclic groups each of order $m$ generated by $r_{i} \ldots r_{n-1} r_{n} r_{n-1} \ldots r_{i}, i=1, \ldots, n$ (respectively).

Define

LeMmA 3.2. Let $k \leqq m$ and $\sigma=\sigma_{1} \ldots \sigma_{k} \in B_{\left(t_{1}, \ldots, t_{k}\right)}^{m}$, where $\sigma_{i} \in B_{t_{i}}^{m}, i=1, \ldots, k$.

$$
\chi_{\left(t_{1}, \ldots, i_{k}\right)}(\sigma)=\xi^{\sum_{i=1}^{k} i f\left(\sigma_{i}\right)},
$$

where $\xi$ is some primitive $m$-th root of unity. Then

(i) $\chi_{\left(t_{1}, \ldots, t_{k}\right)}$ is an irreducible linear representation of $B_{\left(t_{1}, \ldots, t_{k}\right)}^{m}$ and

(ii) $\chi_{\left(t_{1}, \ldots, t_{k}\right)}^{g_{1}}(x) \neq \chi_{\left(t_{1}^{\prime}, \ldots, t_{k}\right)}(x)$ for some $x \in \operatorname{ker} \phi$ and for all $g \in B_{n}^{m}$ unless $\left(t_{1}, \ldots, t_{k}\right)=\left(t_{1}^{\prime}, \ldots, t_{k}^{\prime}\right)$ in which case this holds for all $g \in B_{n}^{m} \backslash B_{\left(t_{1}, \ldots, t_{k}\right)}^{m}$.

(We shall call $\chi_{\left(t_{1}, \ldots, t_{k}\right)}$ the basic linear representation of $B_{\left(t_{1}, \ldots, t_{k}\right)}^{m}$.) 
Proof. (i) Let $\sigma=\sigma_{1} \ldots \sigma_{k}, \sigma^{\prime}=\sigma_{1}^{\prime} \ldots \sigma_{k}^{\prime} \in B_{\left(t_{1}, \ldots, t_{k}\right)}^{m}$ such that $\sigma_{i}, \sigma_{i}^{\prime} \in B_{t_{i}}, \quad i=$ $1, \ldots, k$. Then

$$
\begin{aligned}
\chi_{\left(t_{1}, \ldots, t_{k}\right)}\left(\sigma \sigma^{\prime}\right) & =\chi_{\left(t_{1}, \ldots, t_{k}\right)}\left(\sigma_{1} \ldots \sigma_{k} \sigma_{1}^{\prime} \ldots \sigma_{k}^{\prime}\right) \\
& =\chi_{\left(t_{1}, \ldots, t_{k}\right)}\left(\sigma_{1} \sigma_{1}^{\prime} \ldots \sigma_{k} \sigma_{k}^{\prime}\right) \\
& =\xi^{\sum_{i=1}^{k} f\left(\sigma_{i} \sigma_{i}^{\prime}\right)} \\
& =\xi^{\sum_{i=1}^{k} i f\left(\sigma_{i}\right)+\sum_{i=1}^{k} i f\left(\sigma_{i}^{\prime}\right)} \\
& =\xi^{\sum_{i=1}^{k} i f\left(\sigma_{i}\right)} \xi^{\sum_{k=1}^{k} i f\left(\sigma_{i}^{\prime}\right)} \\
& =\chi_{\left(t_{1}, \ldots, t_{k}\right)}(\sigma) \chi_{\left(t_{1}, \ldots, t_{k}\right)}\left(\sigma^{\prime}\right) .
\end{aligned}
$$

Thus $\chi_{\left(t_{1}, \ldots, t_{k}\right)}$ is a homomorphism from $B_{\left(t_{1}, \ldots, t_{k}\right)}^{m}$ into $\mathbb{C}^{*}$, which proves (i).

(ii) If $\left(t_{1}, \ldots, t_{k}\right) \neq\left(t_{1}^{\prime}, \ldots, t_{k}^{\prime}\right)$, let $i$ be the least index such that $t_{i}^{\prime} \neq t_{i}$. We may assume, without any loss of generality, that $t_{i}<t_{i}^{\prime}$, that is, $P_{i} \subset P_{i}^{\prime}$. If $g \in B_{n}^{m}$ is such that $\phi(g) P_{i}=P_{i}$, let $j \in P_{i}^{\prime} \backslash P_{i}$; then $\phi(g)(j) \in P_{l}, l \neq i$, and we define

$$
x=\left(\begin{array}{cccccc}
1 & 2 & \ldots & j & \ldots & n \\
1 & 2 & \ldots & \xi_{j} & \ldots & n
\end{array}\right)=\left(\begin{array}{c}
j \\
\xi_{j}
\end{array}\right) .
$$

If $\phi(g) P_{i} \neq P_{i}$ then there exists $j \in P_{i} \subset P_{i}^{\prime}$ such that $\phi(g)(j) \in P, 1 \leqq l \leqq k, l \neq i$, and for this $j$ we define $x$ as above. In each case $\chi_{\left(t_{1}^{\prime}, \ldots, t_{k}\right)}(x)=\xi^{i}$, but

$$
\begin{aligned}
\chi_{t_{\left.t_{1}, \ldots, t_{k}\right)}(x)} & =\chi_{\left(t_{1}, \ldots, t_{k}\right)}\left(g x g^{-1}\right) \\
& =\chi_{\left(t_{1}, \ldots, t_{k}\right)}\left(\phi(g) x \phi(g)^{-1}\right) \\
& =\chi_{\left(t_{1}, \ldots, t_{k}\right)}\left(\left(\begin{array}{c}
\phi(g)(j) \\
\xi \phi(g)(j)
\end{array}\right)\right)=\left(\begin{array}{c}
l \\
\xi l
\end{array}\right) \\
& =\xi^{l}, l \neq i .
\end{aligned}
$$

If $\left(t_{1}^{\prime}, \ldots, t_{k}^{\prime}\right)=\left(t_{1}, \ldots, t_{k}\right)$ and $g \in B_{n}^{m} \backslash B_{\left(t_{1}, \ldots, t_{k}\right)}^{m}$ then there exists at least one index $i, 1 \leqq i \leqq k$, and an integer $j \in P_{i}$ such that $\phi(g)(j) \in P_{l}, l \neq i$. Once again we define $x \in \operatorname{ker} \phi$ as above for this particular $j$. Clearly

$$
\chi_{\left(t_{1}, \ldots, t_{k}\right)}^{g_{1}}(x)=\xi^{l} \neq \xi^{i}=\chi_{\left(i_{1}^{i}, \ldots, t_{k}\right)}(x),
$$

which completes the proof.

4. Representations of $B_{n}^{m}$. It is well known that the number of inequivalent irreducible linear representations (henceforth abbreviated as i.l.r.) of a Young subgroup $S_{\left(t_{1}, \ldots, t_{k}\right)}=S_{t_{1}} \times \ldots \times S_{t_{k}}$ is equal to $p\left(t_{1}\right) \ldots p\left(t_{k}\right)$. This enables us to state our main result.

THEOREM 4.1. A full set of inequivalent i.l.r. of $B_{n}^{m}$ is given by

$$
\left\{\left(\chi_{\left(t_{1}, \ldots, t_{m}\right)} \otimes \mathbb{P}\right) \uparrow B_{n}^{m}\right\},
$$

where $\left(t_{1}, \ldots, t_{m}\right)$ ranges over all permissible m-tuples, $\chi_{\left(t_{1}, \ldots, t_{m}\right)}$ is the basic linear 
representation of $B_{\left(t_{1}, \ldots, t_{m}\right)}^{m}$ and $\mathbb{P}$ is an i.l.r. of $B_{\left(t_{1}, \ldots, t_{m}\right)}^{m}$ lifted from an i.l.r. $P$ of $S_{\left(t_{1}, \ldots, t_{m}\right)}$, where $P$ ranges over a complete set of inequivalent i.l.r of $S_{\left(t_{1}, \ldots, t_{m}\right)}$ and $\uparrow$ denotes induction of a representation.

Proof. It is clear from Lemma 2.3 that the cardinality of the above set is equal to the number of inequivalent i.l.r. of $B_{n}^{m}$.

Let $\left(t_{1}, \ldots, t_{m}\right)$ and $\left(t_{1}^{\prime}, \ldots, t_{m}^{\prime}\right)$ be two arbitrary permissible $m$-tuples and $\chi_{\left(t_{1}, \ldots, t_{m}\right)}$, $\chi_{\left(t_{1}^{\prime}, \ldots, t_{m}^{\prime}\right)}$ the basic linear representations of $B_{\left(t_{1}, \ldots, t_{m}\right)}^{m}$ and $B_{\left(t_{1}^{\prime}, \ldots, t_{m}^{\prime}\right)}^{m}$ respectively. Let $\mathbb{P}$ and $\mathbb{P}^{\prime}$ be two i.l.r. of $B_{\left(t_{1}, \ldots, t_{m}\right)}^{m}$ and $B_{\left(t_{1}^{\prime}, \ldots, t_{m}^{\prime}\right)}^{m}$ lifted from the i.l.r. $P$ and $P^{\prime}$ of $S_{\left(t_{1}, \ldots, t_{m}\right)}$ and $S_{\left(t_{1}^{\prime}, \ldots, t_{m}^{\prime}\right)}$ respectively. If $\hat{\psi}, \hat{\psi}^{\prime}, \psi$ and $\psi^{\prime}$ denote the characters of $\mathbb{P}, \mathbb{P}^{\prime}, P$ and $P^{\prime}$ respectively, then we prove that

$$
\left(\left(\chi_{\left(t_{1}, \ldots, t_{m}\right)} \hat{\psi}\right) \uparrow B_{n}^{m},\left(\chi_{\left(t^{\prime}, \ldots, t_{m}^{\prime}\right)} \hat{\psi}^{\prime}\right) \uparrow B_{n}^{m}\right)_{B_{n}^{m}}=0
$$

unless $\left(t_{1}, \ldots, t_{m}\right)=\left(t_{1}^{\prime}, \ldots, t_{m}^{\prime}\right)$ and $\psi=\psi^{\prime}$ in which case it is equal to 1 . This will complete the proof of the theorem.

By Frobenius' reciprocity theorem and Mackey's subgroup theorem, the above inner product is equal to

$$
\begin{aligned}
& \left(\left(\chi_{\left(t_{1}, \ldots, t_{m}\right)} \hat{\psi}\right),\left(\left(\left(\chi_{\left(t_{1}^{\prime}, \ldots, t_{m}^{\prime}\right)} \hat{\psi}^{\prime}\right) \uparrow B_{n}^{m}\right) \downarrow B_{\left(t_{1}, \ldots, t_{m}\right)}^{m}\right)\right)_{B_{\left(t_{1}, \ldots, t_{m}\right)}^{m}} \\
& \quad=\sum_{x}\left(\left(\chi_{\left(t_{1}, \ldots, t_{m}\right)} \hat{\psi}\right),\left(\left(\chi_{\left(t_{1}^{\prime}, \ldots, t_{m}^{\prime}\right)} \hat{\psi}^{\prime}\right)^{x} \downarrow H_{x}\right) \uparrow B_{\left(t_{1}, \ldots, t_{m}\right)}^{m}\right)_{B_{\left(t_{1}, \ldots, t_{m}\right)}^{m}} \\
& \quad=\sum_{x}\left(\left(\chi_{\left(t_{1}, \ldots, t_{m}\right)} \hat{\psi}\right) \downarrow H_{x},\left(\chi_{\left(t_{1}^{\prime}, \ldots, t_{m}^{\prime}\right)} \hat{\psi}^{\prime}\right) \downarrow H_{x}\right)_{H_{x}},
\end{aligned}
$$

where $H_{x}=B_{\left(t_{1}, \ldots, t_{m}\right)}^{m} \cap x^{-1} B_{\left(t_{1}^{\prime}, \ldots, t_{m}^{\prime}\right)}^{m} x$ and $x$ ranges over all representative elements of a double coset decomposition of $B_{n}^{m}$ relative to the generalized Young subgroups $B_{\left(t_{1}, \ldots, t_{m}\right)}^{m}$ and $B_{\left(t_{1}^{\prime}, \ldots, t_{m}^{\prime}\right)}^{m}$.

We claim that each of the terms in the above summation is zero except in the case noted earlier. For, if for some $x$

$$
\left(\chi_{\left(t_{1}, \ldots, t_{m}\right)} \hat{\psi}\right) \downarrow H_{x} \text { and }\left(\chi_{\left(r_{1}^{\prime}, \ldots, t_{m}^{\prime}\right)} \hat{\psi}^{\prime}\right)^{x} \downarrow H_{x}
$$

have an irreducible component in common then so do

$$
\left(\chi_{\left(t_{1}, \ldots, t_{m}\right)} \psi\right) \downarrow \operatorname{ker} \phi \quad \text { and } \quad\left(\chi_{\left(t_{1}^{\prime}, \ldots, t_{m}^{\prime}\right)} \psi^{\prime}\right)^{x} \downarrow \operatorname{ker} \phi .
$$

(Note that ker $\phi \subseteq B_{\left(t_{1}, \ldots, t_{m}\right)}^{m} \cap x^{-1} B_{\left(t_{1}^{\prime}, \ldots, t_{m}^{\prime}\right)}^{m} x$ for all $x$.)

But in this case these representations are multiples of

$$
\chi_{\left(t_{1}, \ldots, t_{m}\right)} \downarrow \operatorname{ker} \phi \quad \text { and } \quad \chi_{\left(t_{1}^{\prime}, \ldots, t_{m}^{\prime}\right)}^{x} \downarrow \operatorname{ker} \phi
$$

respectively. Both of these representations being irreducible, we get

$$
\chi_{\left(t_{1}, \ldots, t_{m}\right)}=\chi_{\left(r_{1}^{\prime}, \ldots, t_{m}^{\prime}\right)}^{x} \text { on } \operatorname{ker} \phi .
$$

By Lemma 3.2, this implies that

$$
\left(t_{1}, \ldots, t_{m}\right)=\left(t_{1}^{\prime}, \ldots, t_{m}^{\prime}\right) \text { and } x \in B_{\left(t_{1}, \ldots, t_{m}\right)}^{m} .
$$


Thus the possibility of getting a nonzero term arises from the inner product

$$
\left(\chi_{\left(t_{1}, \ldots, t_{m}\right)} \hat{\psi}, \chi_{\left(t_{1}, \ldots, t_{m}\right)} \hat{\psi}^{\prime}\right)_{B_{\left(t_{1}, \ldots,,_{m}\right)}^{m}}
$$

which is equal to

$$
\begin{aligned}
& \frac{1}{\left|B_{\left(t_{1}, \ldots, t_{m}\right)}^{m}\right|} \sum_{\sigma \in B_{\left(t_{1}, \ldots, t_{m}\right)}^{m}}\left(\chi_{\left(t_{1}, \ldots, t_{m}\right)} \hat{\psi}\right)(\sigma) \overline{\left(\chi_{\left(t_{1}, \ldots, t_{m}\right)} \hat{\psi}^{\prime}\right)(\sigma)} \\
& =\frac{1}{\left|B_{\left(t_{1}, \ldots, t_{m}\right)}^{m}\right|_{\sigma \in B_{\left(t_{1}, \ldots, t_{m}\right)}^{m}}} \chi_{\left(t_{1}, \ldots, t_{m}\right)}(\sigma) \overline{\chi_{\left(t_{1}, \ldots, t_{m}\right)}(\sigma)} \hat{\psi}(\sigma) \overline{\hat{\psi}^{\prime}(\sigma)} \\
& =\frac{1}{\left|B_{\left(t_{1}, \ldots, t_{m}\right)}^{m}\right|} \sum_{\sigma \in B_{\left(t_{1}, \ldots, r_{m}\right)}^{m}} \psi(\sigma) \overline{\psi(\sigma)} \\
& \text { (since } \chi_{\left(t_{1}, \ldots, t_{m}\right)}(\sigma) \overline{\chi_{\left(t_{1}, \ldots, t_{m}\right)}(\sigma)}=\left|\chi_{\left(t_{1}, \ldots, t_{m}\right)}(\sigma)\right|^{2}=1 \text { ) } \\
& \begin{array}{l}
=\frac{1}{\mid S_{\left(t_{1}, \ldots, I_{m}\right)}} \sum_{\sigma_{1} \in S_{\left(t_{1}, \ldots, I_{m}\right)}} \psi\left(\sigma_{1}\right) \overline{\psi^{\prime}\left(\sigma_{1}\right)} \\
=\left(\psi, \psi^{\prime}\right)
\end{array}
\end{aligned}
$$

and this is nonzero if and only if $\psi=\psi^{\prime}$, in which case it is equal to 1 because $\psi$ and $\psi^{\prime}$ are both irreducible.

Acknowledgment. I wish to thank Professor A. O. Morris for his advice and guidance and the Government of Pakistan for supporting this work.

\section{REFERENCES}

1. H. S. M. Coxeter, The abstract groups $R^{m}=S^{m}=\left(R^{i} S^{i}\right)^{p_{i}}=1, S^{m}=T^{2}=\left(S^{i} T\right)^{2 p_{i}}=1$, and $S^{m}=T^{2}=\left(S^{-j} T S^{i} T\right)^{p_{j}}=1$, Proc. London Math. Soc. 41 (1936), 278-301.

2. A. Kerber, Representations of Permutations Groups I, Lecture Notes in Mathematics, No. 240 (Springer, 1971).

3. M. Osima, On the representations of the generalized symmetric groups, Math. J. Okayama Univ. 4 (1954), 39-56.

4. B. M. Puttaswamaiah, Unitary representations of generalized symmetric groups, Canad. J. Math. 21 (1969), 28-38.

5. E.W. Read, On the $\alpha$-regular classes of the generalised symmetric group, Glasgow Math. J. 17 (1976), 144-150.

6. M. Saeed-ul-Islam, Irreducible projective representations of the generalized symmetric group $B_{n}^{m}$, Proc. XII international Colloq. on Group Theoretical Methods in Physics, Lecture Notes in Physics No. 201 (Springer, 1983), 70-72.

Department of Pure Mathematics UNIVERSITY COLLEGE OF WALES ABERYSTWYTH, U.K.
Present address:

Department of Mathematics BAyERo UNIVERSITY PMB 3011, KaNo, Nigeria 